Research Article

\title{
Cardy-Verlinde Formula of Noncommutative Schwarzschild Black Hole
}

\author{
G. Abbas \\ Department of Mathematics, COMSATS Institute of Information Technology, Sahiwal 57000, Pakistan \\ Correspondence should be addressed to G. Abbas; abbasg91@yahoo.com
}

Received 19 November 2013; Revised 3 February 2014; Accepted 13 February 2014; Published 18 March 2014

Academic Editor: George Siopsis

Copyright (C) 2014 G. Abbas. This is an open access article distributed under the Creative Commons Attribution License, which permits unrestricted use, distribution, and reproduction in any medium, provided the original work is properly cited. The publication of this article was funded by SCOAP

\begin{abstract}
Few years ago, Setare (2006) has investigated the Cardy-Verlinde formula of noncommutative black hole obtained by noncommutativity of coordinates. In this paper, we apply the same procedure to a noncommutative black hole obtained by the coordinate coherent approach. The Cardy-Verlinde formula is entropy formula of conformal field theory in an arbitrary dimension. It relates the entropy of conformal field theory to its total energy and Casimir energy. In this paper, we have calculated the total energy and Casimir energy of noncommutative Schwarzschild black hole and have shown that entropy of noncommutative Schwarzschild black hole horizon can be expressed in terms of Cardy-Verlinde formula.
\end{abstract}

\section{Introduction}

Verlinde [1] proved that the entropy of conformal field theory in arbitrary dimension is related to its total energy and Casimir energy; this is known as generalized Verlinde formula (commonly termed as Cardy-Verlinde formula). Recently, it has been investigated that this formula holds well for Reissner-Nordström de-Sitter black hole (BH) [2] and charged Kerr BH [3]. Birmingham and Mokhtari proved the validity of Birmingham and Mokhtari [4] proved the Verlinde formula for Taub-Bolt-Anti-de-Sitter BH. Setare and Jamil [5] discussed the Cardy-Verlinde formula for charged BTZ BH. Many authors [6-11] have proved the validity of CardyVerlinde for different BHs. The purpose of this paper is to investigate the validity of Cardy-Verlinde entropy formula for NC Schwarzschild BH.

In classical general relativity (GR), the curvature singularity is such a point where physical description of the gravitational field is impossible. This problem can be removed in GR by taking into account the quantum mechanical treatment to the standard formulation of GR. Motivated by such reasoning, some $\mathrm{BH}$ solutions in noncommutative (NC) field theory have been derived. In these solutions, curvature singularity at origin is removed by de-Sitter core which is introduced due to NC nature of spacetime [12]. Ansoldi et al. [13] formulated the NC charged BHs solutions; this was extended to rotating noncommutative $\mathrm{BHs}$ case by Modesto and Nicolini [14]. Mann and Nicoloni [15] have discussed the cosmological production of NC BHs. The first NC version of wormholes solution was investigated by Nicolini and Spallucci [16]. Farook et al. [17] have investigated the higher dimensional wormhole solutions in NC theory of gravity. Motivated by such NC correction to BHs, Sharif and Abbas [18] studied the thin shell collapse in NC Reissner-Nordström geometry. Banerjee and Gangopadhyay [19] derived the Komar energy and Sammar formula for NC Schwarzschild $\mathrm{BH}$.

Motivated by the recent development in NC theory of gravity, we have proved that the entropy of NC Schwarzschild $\mathrm{BH}$ horizon can be expressed in terms of Cardy-Verlinde formula. For this purpose, we have used the Setare and Jamil method [5]. The plan of the paper is as follows. In Section 2, we briefly discussed the the thermodynamical relations of NC Schwarzschild $\mathrm{BH}$ and Cardy-Verlinde formula and proved that entropy of noncommutative Schwarzschild $\mathrm{BH}$ horizon can be expressed in terms of Cardy-Verlinde formula. Section 3 is devoted to the concluded remarks of the work done. 


\section{Noncommutative Schwarzschild Black Hole and Cardy-Verlinde Formula}

According to GR, singularity is such a region of spacetime at which the usual laws of physics break down. This problem can be removed by applying the formulation of NC field theory to GR. For example, the NC BHs are one of the outcomes of string theory. These have such geometric structure in which curvature singularity is recovered by the minimal length introduced by the NC nature of coordinates. Further, all types of NC BHs expose the de-Sitter core due to quantum fluctuations at the center of the manifold.

The NC formulation of GR is one of the long standing problems which hava no solution yet. An extensive literature survey [20-22] implies that the application of Moyal $\star-$ product among the tetrad fields in the gravitational action is a mathematically correct approach but not physically. It is due to the fact that the expansion of $\star$-product in NC parameter is truncated up to a desirable order which causes destruction of the nonlocal nature of NC theory. This result is to face the $\mathrm{BH}$ geometry with the same curvature singularities as in GR. Instead of using $\star$-product, one can formulate NC form of GR using the coordinate coherent state approach.

In this approach, the density of point like source in NC spacetime can be governed by a Gaussian distribution by using the relation [12]

$$
\rho=\frac{M e^{-r^{2} / 4 \Theta}}{(4 \pi \Theta)^{3 / 2}},
$$

where $M$ is constant gravitational mass and $\Theta$ is constant having the dimension of length squared. The line element for NC Schwarzschild $\mathrm{BH}$ is [12]

$$
d s^{2}=f(r) d t^{2}-\frac{1}{f(r)} d r^{2}-r^{2}\left(d \theta^{2}+\sin ^{2} \theta d \phi^{2}\right),
$$

where $f(r)=1-(4 M / r \sqrt{\pi}) \gamma\left(3 / 2 ; r^{2} / 4 \Theta\right)$ and $\gamma$ is lower incomplete gamma function which is defined by

$$
\gamma\left(\frac{a}{b} ; x\right)=\int_{0}^{x} t^{a / b-1} e^{-t} d t
$$

In the commutative limit $r / \sqrt{\Theta} \rightarrow \infty$, that is, $\Theta \rightarrow 0$, (2) reduces to conventional Schwarzschild metric. The event horizons of $\mathrm{BH}$ can be found by setting $f\left(r_{h}\right)=0$, which yields

$$
r_{h}=\frac{4 M}{\sqrt{\pi}} \gamma\left(\frac{3}{2} ; \frac{r_{h}^{2}}{4 \Theta}\right)
$$

We take the large radius regime $\left(r_{h}^{2} / 4 \Theta \gg 1\right)$ where we can expand the incomplete gamma function to solve $r_{h}$ by iteration. By keeping the terms up to order $\sqrt{\Theta} e^{-M^{2} / \Theta}$, we find

$$
r_{h} \simeq 2 M\left[1-\frac{2 M}{\sqrt{\pi \Theta}}\left(1+\frac{\Theta}{2 M^{2}}\right) e^{-M^{2} / \Theta}\right] .
$$

Now the Hawking temperature for NC Schwarzschild BH up to order $\sqrt{\Theta} e^{-M^{2} / \Theta}$ is given by

$$
T_{H}=\frac{1}{8 \pi M}\left[1-\frac{4 M^{3}}{\Theta \sqrt{\pi \Theta}}\left(1-\frac{\Theta}{2 M^{2}}-\frac{\Theta^{2}}{4 M^{4}}\right) e^{-M^{2} / \Theta}\right] .
$$

The entropy of the NC Schwarzschild BH $\left(S=A / 4=\pi r_{h}^{2}\right)$ up to order $\sqrt{\Theta} e^{-M^{2} / \Theta}$ is given by

$$
S=4 \pi M^{2}\left[1-\frac{4 M}{\sqrt{\pi \Theta}}\left(1+\frac{\Theta}{2 M^{2}}\right)\right] e^{-M^{2} / \Theta} .
$$

The generalized form of Cardy formula (also known as Cardy-Verlinde formula) is given by [5]

$$
S_{\mathrm{CFT}}=\frac{2 \pi R}{\sqrt{a b}} \sqrt{E_{C}\left(2 E-E_{\mathrm{C}}\right)}
$$

where $a, b>0, R$ is radius of $n+1$-dimensional FRW universe, $E_{C}$ is the Casimir energy, and $E$ is the total energy of underlying field. The definition of Casimir energy is derived by the violation of Euler's relation as [6]

$$
E_{C}=n(E+P V-T S-\Phi Q-\Omega J),
$$

where the pressure for CFT is $P=E / n V, J$ and $Q$ are zero for NC Schwarzschild $\mathrm{BH}$, and $V$ is the volume of the system bounded by the apparent horizon. The total energy may be written as sum of extensive part $E_{E}$ and Casimir energy $E_{C}$ as

$$
E=E_{E}+\frac{1}{2} E_{C}
$$

The Casimir energy $E_{C}$ as well as purely extensive part of energy $E_{E}$ can be expressed in terms of entropy $S$ and radius $R$

$$
\begin{aligned}
& E_{E}=\frac{a}{4 \pi R} S^{1+1 / n} ; \\
& E_{C}=\frac{b}{2 \pi R} S^{1-1 / n} .
\end{aligned}
$$

After the work of Witten [23] on the $\mathrm{AdS}_{d} / \mathrm{CFT}_{d-1}$ correspondence, Savonije and Verlinde [24] proposed that CardyVerlinde formula can be derived using the thermodynamical relations of arbitrary BHs in arbitrary dimensions. In this point of view, we shall prove the validity of Cardy-Verlinde formula for NC Schwarzschild BH.

From (8) and (10), we get

$$
S_{\mathrm{CFT}}=\frac{2 \pi R}{\sqrt{a b}} \sqrt{2 E_{E} E_{C}}
$$

By using (11) and (12) in the above equation,

$$
S_{\mathrm{CFT}}=S \text {. }
$$

The Casimir energy given by (9) for $n=2$ with (6) and (7) takes the following:

$$
\begin{aligned}
E_{C}= & 3 E-2 T S, \\
= & 3 E-M\left[1-\frac{4 M^{3}}{\Theta \sqrt{\pi \Theta}}\left(1-\frac{\Theta}{2 M^{2}}-\frac{\Theta^{2}}{4 M^{4}}\right) e^{-M^{2} / \Theta}\right] \\
& \times\left[1-\frac{4 M}{\sqrt{\pi \Theta}}\left(1+\frac{\Theta}{2 M^{2}}\right) e^{-M^{2} / \Theta}\right] .
\end{aligned}
$$


By using the above equation in (10), we get the purely extensive part of total energy as

$$
\begin{aligned}
E_{E}= & -\frac{E}{2}+T S \\
= & -\frac{E}{2}+\frac{M}{2}\left[1-\frac{4 M^{3}}{\Theta \sqrt{\pi \Theta}}\left(1-\frac{\Theta}{2 M^{2}}-\frac{\Theta^{2}}{4 M^{4}}\right) e^{-M^{2} / \Theta}\right] \\
& \times\left[1-\frac{4 M}{\sqrt{\pi \Theta}}\left(1+\frac{\Theta}{2 M^{2}}\right) e^{-M^{2} / \Theta}\right]
\end{aligned}
$$

Further,

$$
\begin{aligned}
2 E-E_{C}= & E+2 T S \\
= & -E \\
& +M\left[1-\frac{4 M^{3}}{\Theta \sqrt{\pi \Theta}}\left(1-\frac{\Theta}{2 M^{2}}-\frac{\Theta^{2}}{4 M^{4}}\right) e^{-M^{2} / \Theta}\right] \\
& \times\left[1-\frac{4 M}{\sqrt{\pi \Theta}}\left(1+\frac{\Theta}{2 M^{2}}\right) e^{-M^{2} / \Theta}\right] .
\end{aligned}
$$

From the comparison of (12) and (16), we get

$$
\begin{aligned}
R= & \frac{b S^{1 / 2}}{4 \pi}\left(\frac{3}{2} E-T S\right)^{-1} \\
= & \frac{b M}{\sqrt{4 \pi}}\left[1-\frac{4 M}{\sqrt{\pi \Theta}}\left(1+\frac{\Theta}{2 M^{2}}\right) e^{-M^{2} / \Theta}\right]^{1 / 2} \\
\times\left(\frac{3}{2} E-\frac{M}{2}\right. & \\
\times & {\left[1-\frac{4 M^{3}}{\Theta \sqrt{\pi \Theta}}\left(1-\frac{\Theta}{2 M^{2}}-\frac{\Theta^{2}}{4 M^{4}}\right) e^{-M^{2} / \Theta}\right] } \\
\times & {\left.\left[1-\frac{4 M}{\sqrt{\pi \Theta}}\left(1+\frac{\Theta}{2 M^{2}}\right) e^{-M^{2} / \Theta}\right]\right)^{-1} . }
\end{aligned}
$$

Also, the comparison of (11) and (18) gives

$$
\begin{aligned}
R=\frac{a S^{3 / 2}}{4 \pi} & \left(-\frac{1}{2} E+T S\right)^{-1} \\
= & 4 \pi a M^{3}\left[1-\frac{4 M}{\sqrt{\pi \Theta}}\left(1+\frac{\Theta}{2 M^{2}}\right) e^{-M^{2} / \Theta}\right]^{3 / 2} \\
\times & \left(-\frac{1}{2} E\right. \\
+ & \frac{M}{2}\left[1-\frac{4 M^{3}}{\Theta \sqrt{\pi \Theta}}\left(1-\frac{\Theta}{2 M^{2}}-\frac{\Theta^{2}}{4 M^{4}}\right) e^{-M^{2} / \Theta}\right] \\
& \left.\times\left[1-\frac{4 M}{\sqrt{\pi \Theta}}\left(1+\frac{\Theta}{2 M^{2}}\right) e^{-M^{2} / \Theta}\right]\right)^{-1} .
\end{aligned}
$$

By taking the product of (20) and (22), we get

$$
R=\frac{\sqrt{a b}}{4 \pi} \frac{S}{\sqrt{(-(1 / 2) E+T S)((3 / 2) E-T S)}} .
$$

By using (16), (18), and (24) in (8), we get

$$
S_{\mathrm{CFT}}=S \text {. }
$$

This result shows that the entropy of the NC Schwarzschild $\mathrm{BH}$ can be expressed in terms of Cardy-Verlinde formula. As the $\mathrm{BH}$ geometric and thermodynamic quantities are evaluated by assuming large-radius approximations, the CardyVerlinde formula is valid only for large BHs.

\section{Outlook}

As a prolongation of the research on $\mathrm{BH}$ and gravitational collapse [12-31] in this paper, we derive the entropy formula in conformal field theory of a 4D static spherically symmetric NC Schwarzschild BH. This NC BH solution is obtained by introducing the NC effect through a coordinate coherent state approach, which is in fact the substitution of the point distributions by smeared source throughout a regular region of linear size. We perform the analysis by obtaining entropy and temperature, which show a deviation from their usual relations depending on the NC parameter $\Theta$. We have proved that the entropy of the NC Schwarzschild $\mathrm{BH}$ can be expressed in terms of Cardy-Verlinde formula. For this purpose, we have used the approximate of values of incomplete gamma functions up to the term $\sqrt{\Theta} e^{-M^{2} / \Theta}$. With the same order of approximation the entropy and temperature of $\mathrm{NC} \mathrm{BH}$ horizons has been calculated. The procedure adopted in this paper has been already used by Setare and Jamil $[5,6]$. It would be interesting to generalize this work for charged and charged rotating NC BHs. The Cardy-Verlinde formula of charged $\mathrm{NC} \mathrm{BH} \mathrm{[32]} \mathrm{is} \mathrm{in} \mathrm{progress.}$

\section{Conflict of Interests}

The author declares that there is no conflict of interests regarding the publication of this paper.

\section{References}

[1] E. Verlinde, "On the holographic principle in a radiation dominated universe," http://arxiv.org/abs/hep-th/0008140.

[2] M. R. Setare, "The Cardy-Verlinde formula and entropy of topological Reissner-Nordström black holes in de Sitter spaces," Modern Physics Letters A, vol. 17, no. 32, pp. 2089-2094, 2002.

[3] M. R. Setare and M. B. Altaie, "The Cardy-Verlinde formula and entropy of topological Kerr-Newman black holes in de Sitter spaces," The European Physical Journal C, vol. 30, no. 2, pp. 273277, 2003.

[4] D. Birmingham and S. Mokhtari, "The Cardy-Verlinde formula and Taub-bolt-AdS spacetimes," Physics Letters B, vol. 508, no. 3-4, pp. 365-368, 2001.

[5] M. R. Setare and M. Jamil, "The Cardy-Verlinde formula and entropy of the charged rotating BTZ black hole," Physics Letters $B$, vol. 681, no. 5, pp. 469-471, 2009.

[6] M. R. Setare and M. Jamil, "Cardy-Verlinde formula of Kehagias-Sfetsos black hole," International Journal of Theoretical Physics, vol. 50, no. 2, pp. 511-518, 2011.

[7] M. R. Setare and R. Mansouri, "Holographic thermodynamics on the brane in topological Reissner-Nordström de Sitter space," 
International Journal of Modern Physics A, vol. 18, no. 24, pp. 4443-4450, 2003.

[8] M. R. Setare and E. C. Vagenas, "Cardy-Verlinde formula and Achúcarro-Ortiz black hole," Physical Review D, vol. 68, no. 6, Article ID 064014, 5 pages, 2003.

[9] M. R. Setare and E. C. Vagenas, "Self-gravitational corrections to the Cardy-Verlinde formula and the FRW brane cosmology in $S_{d S_{5}}$ bulk," International Journal of Modern Physics A, vol. 20, no. 30, pp. 7219-7232, 2005.

[10] M. R. Setare, "Logarithmic correction to the Cardy-Verlinde formula in Achúcarro-Oritz black hole," The European Physical Journal C, vol. 33, no. 4, pp. 555-559, 2004.

[11] B. Wang, E. Abdalla, and R.-K. Su, "Relating Friedmann equation to Cardy formula in universes with cosmological constant," Physics Letters B, vol. 503, no. 3-4, pp. 394-398, 2001.

[12] P. Nicolini, A. Smailagic, and E. Spallucci, "Noncommutative geometry inspired Schwarzschild black hole," Physics Letters B, vol. 632, no. 4, pp. 547-551, 2006.

[13] S. Ansoldi, P. Nicolini, A. Smailagic, and E. Spallucci, "Noncommutative geometry inspired charged black holes," Physics Letters B, vol. 645, no. 2-3, pp. 261-266, 2007.

[14] L. Modesto and P. Nicolini, "Charged rotating noncommutative black holes," Physical Review D, vol. 82, no. 10, Article ID 104035, 14 pages, 2010.

[15] R. B. Mann and P. Nicoloni, "Cosmological production of noncommutative black holes," Physical Review D, vol. 84, no. 6, Article ID 064014, 13 pages, 2011.

[16] P. Nicolini and E. Spallucci, "Noncommutative geometry-inspired dirty black holes," Classical and Quantum Gravity, vol. 27, no. 1, Article ID 015010, 10 pages, 2010.

[17] R. Farook, S. Islam, P. K. F. Kuhfittig, and S. Ray, "Searching for higher-dimensional wormholes with noncommutative geometry," Physical Review D, vol. 86, no. 10, Article ID 106010, 7 pages, 2012.

[18] M. Sharif and G. Abbas, "Non-commutative correction to thin shell collapse in Reissner-Nordstrom geometry," Journal of the Physical Society of Japan, vol. 81, no. 4, Article ID 044002, 2012.

[19] R. Banerjee and S. Gangopadhyay, "Komar energy and Smarr formula for noncommutative inspired Schwarzschild black hole," General Relativity and Gravitation, vol. 43, no. 11, pp. 32013212, 2011.

[20] A. Smailagic and E. Spallucci, "Feynman path integral on the non-commutative plane," Journal of Physics A, vol. 36, no. 33, pp. L467-L471, 2003.

[21] A. Smailagic and E. Spallucci, "UV divergence-free QFT on noncommutative plane," Journal of Physics A, vol. 36, no. 39, pp. L517-L521, 2003.

[22] A. Smailagic and E. Spallucci, "Lorentz invariance, unitarity and UV-finiteness of QFT on noncommutative spacetime," Journal of Physics A, vol. 37, no. 28, p. 1, 2004, Erratum in Journal of Physics A, vol. 37, no. 28, pp. 7169-7178, 2004.

[23] E. Witten, "Anti-de Sitter space, thermal phase transition, and confinement in gauge theories," Advances in Theoretical and Mathematical Physics, vol. 2, no. 3, pp. 505-532, 1998.

[24] I. Savonije and E. Verlinde, "CFT and entropy on the brane," Physics Letters B, vol. 507, no. 1-4, pp. 305-311, 2001.

[25] R. Banerjee, S. Gangopadhyay, and S. K. Modak, "Voros product, noncommutative Schwarzschild black hole and corrected area law," Physics Letters B, vol. 686, no. 2-3, pp. 181-187, 2010.

[26] R. Banerjee, B. R. Majhi, and S. Samanta, "Noncommutative black hole thermodynamics," Physical Review D, vol. 77, no. 12, Article ID 124035, 8 pages, 2008.
[27] R. Banerjee, B. R. Majhi, and S. K. Modak, "Noncommutative Schwarzschild black hole and area law," Classical and Quantum Gravity, vol. 26, no. 8, Article ID 085010, 11 pages, 2009.

[28] M. Sharif and G. Abbas, "Dynamics of shearfree dissipative collapse in $f(G)$ gravity," Journal of the Physical Society of Japan, vol. 82, Article ID 034006, 6 pages, 2013.

[29] M. Sharif and G. Abbas, "Dynamics of charged radiating collapse in modified Gauss-Bonnet gravity," The European Physical Journal Plus, vol. 128, p. 102, 2013.

[30] M. Sharif and G. Abbas, "Singularities of noncompact charged objects," Chinese Physics B, vol. 22, Article ID 030401, 2013.

[31] G. Abbas and R. M. Ramzan, "Thermodynamics of phantom energy accreting onto a black hole in Einstein-power-Maxwell gravity," Chinese Physics Letters, vol. 30, no. 10, Article ID 100403, 2013.

[32] G. Abbas, "Cardy-Verlinde formula of non-commutative chargedBH," under progress. 

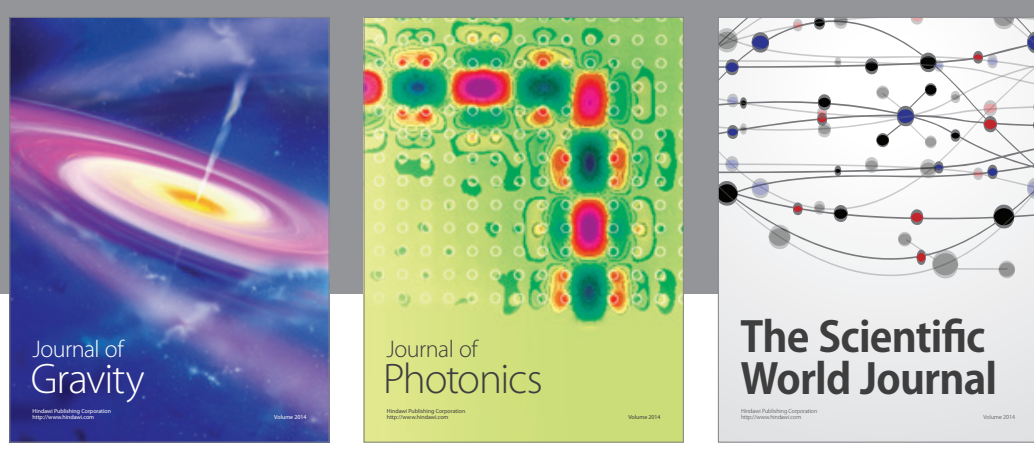

The Scientific World Journal
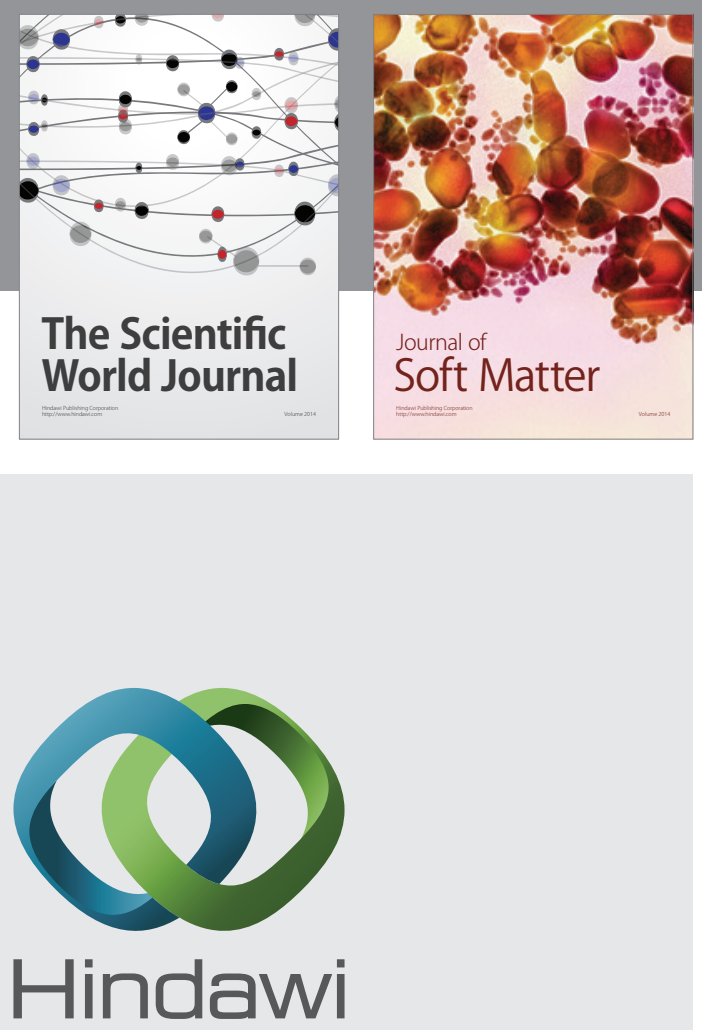

Submit your manuscripts at

http://www.hindawi.com

nternational Journal of

Statistical Mechanics
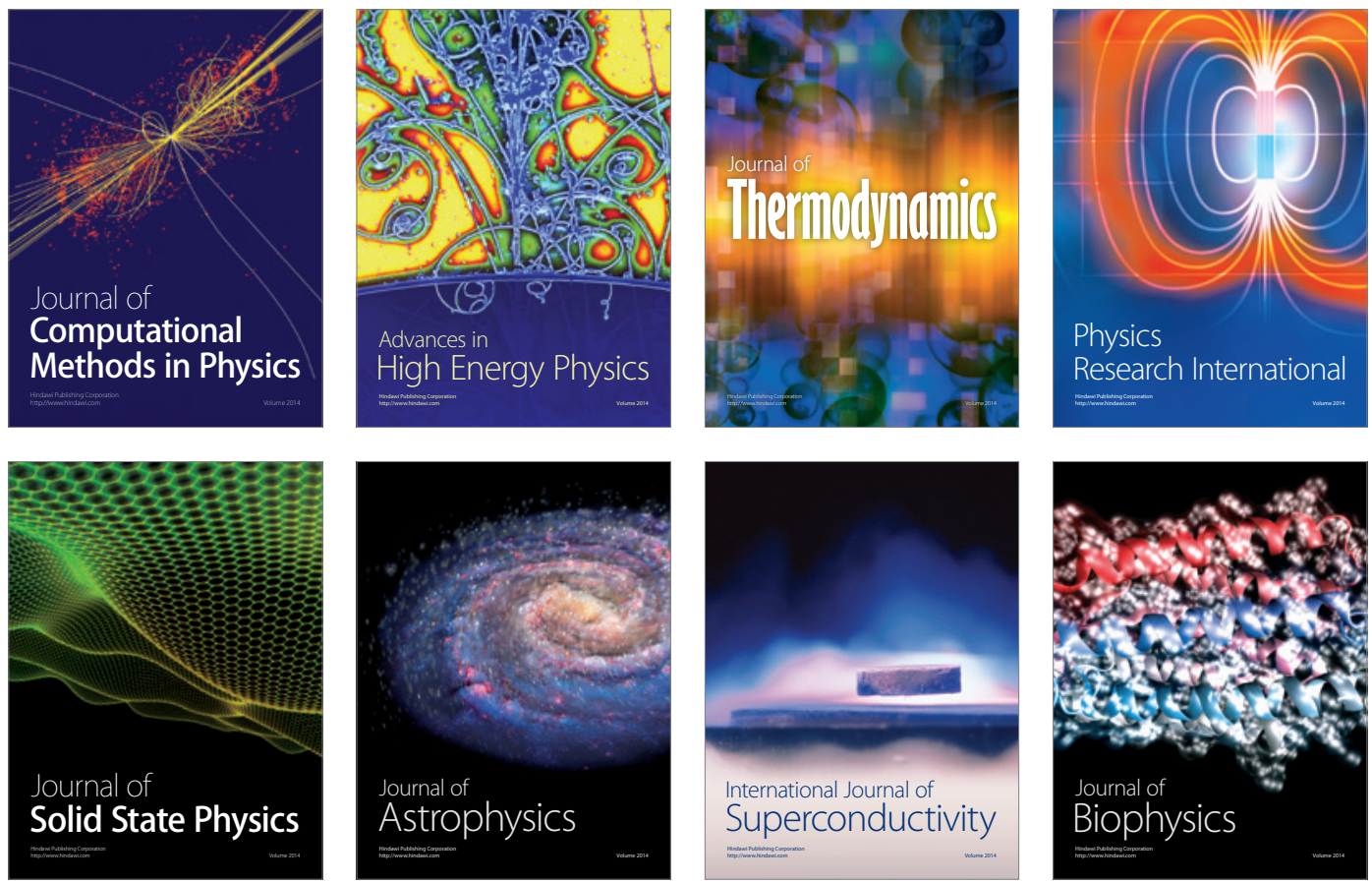
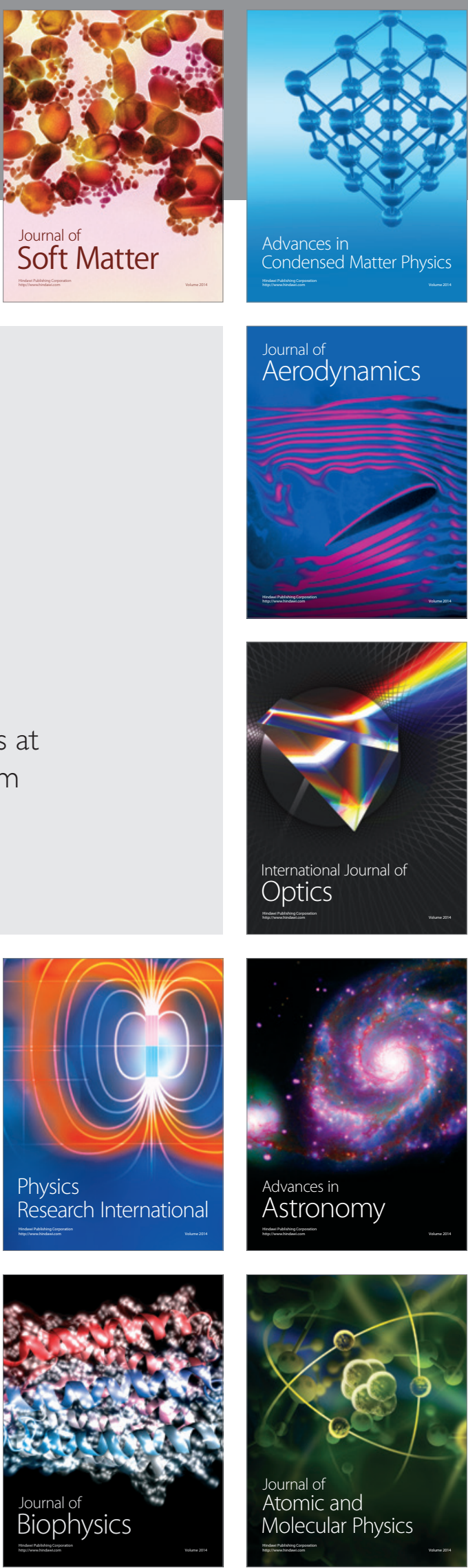